\title{
Presentación Dossier
}

\author{
ACONTECIMIENTO, EMERGENCIA \\ Y DISCONTINUIDAD EN LA HISTORIA. \\ (LOS PORQUÉS DE UN DOSSIER DE TEORÍA DE LA HISTORIA HOY)
}

Pablo Aravena Núñez*

François Hartog, en un texto titulado "La temporalización del tiempo" (2011)1, plantea la tesis acerca de la existencia de una cierta continuidad entre la función social que desempeñaba el profeta en el mundo judío antiguo y la que desempeña el historiador en las sociedades modernas. Son -o eran- ante todo quienes articulan el tiempo. Es a ellos quienes se interroga ante el impacto de un acontecimiento devastador, esto es, tal como lo ha definido LaCapra "que supera la capacidad imaginativa de concebirlo o anticiparlo". Sus respuestas tienen que ver con un reordenamiento de los hechos del pasado para dar cabida a un nuevo acontecimiento que impacta y así restituir el sentido de una trama que de pronto se ha vuelto ya inverosímil. El profeta, entonces, señala Hartog, ante todo trabaja con el pasado y no con el futuro, como usualmente suponemos. Pero luego efectúa un oscuro pronóstico (profecía) del futuro, para finalizar con la advertencia de un: "a menos que hoy tomemos la decisión adecuada [...]". El profeta no está obligado a "no fallar" respecto de lo afirmado sobre el futuro, pues su obligación es ante todo respecto del presente, este es su tiempo, su deber es propiciarlo como el instante de la decisión correcta, es quien promueve la acción colectiva, por ello su eficacia se confirma cuanto más falla su profecía; es decir, cuanto más logra alejar a su pueblo de aquel oscuro futuro predicado. Pero antes que esto, el profeta "salva" a su pueblo por el solo hecho de interpretar el acontecimiento restituyendo y asegurando un sentido. En ambos casos el pueblo es salvado "falsamente". ¿Por qué comenzar

* Profesor titular del Instituto de Historia y Ciencias Sociales de la Universidad de Valparaíso, Chile. Doctor en Estudios Latinoamericanos por la Universidad de Chile. ORCID ID: https:// orcid.org/0000-0001-7373-4265.Correo electrónico: pablo.aravena@uv.cl

1 Hartog, 2011, pp. 13-33. 
señalando esto? Porque buena parte de la operación descrita coincide con lo que modernamente se designó como "conciencia histórica".

Pese a que la palabra historia es un invento griego, nunca significó estudio del pasado, sino tan solo "indagación en base a testigos", por lo que tiene más que ver con el germen del método historiador que con nuestra idea sobre la estructura del acontecer mismo. Esta es irrenunciablemente una herencia judeocristiana (con todo lo criticable que puede ser este sintagma).

Cuando Agustín de Hipona debió responder ante la perplejidad y las acusaciones contra el cristianismo que se desprendían de ese otro acontecimiento devastador que fue la invasión bárbara al corazón del imperio romano apenas convertida Roma al cristianismo (411 d.C.), procedió según la tradición profética antigua: si el acontecimiento no se comprendía lo era únicamente por la falta de fe, condición esta que determinaba explicarlo solo en relación con los acontecimientos más próximos en el tiempo, más bien con los del pasado reciente (tal como habían procedido desde el origen de la historiografía los historiadores griegos y sus émulos romanos). Entonces la exigencia de Agustín, de una renovada fe, era también la exigencia de otro concepto de tiempo e historia: de la consideración de otros acontecimientos y de unas nuevas relaciones entre ellos: ahora, para comprender, había que retroceder a tiempos remotos, solo abarcables por una historia sagrada que hacía suya la historia judía. Cuanto más profundo el tiempo en que se reordenaban los acontecimientos, más sólido el lugar que se conseguía para re-inscribir el nuevo acontecimiento que había detonado la crisis. Solo en esta tradición el pasado podía tener futuro.

Esta tradición antigua engendró - expansión colonial europea mediante- la que había sido hasta aquí "nuestra" tradición: la tradición de la Historia². Pero como lo planteara hace ya tiempo Lévi-Strauss, se trata tan solo de un modo posible de existir. Los pueblos "de la Historia" integran y potencian el acontecimiento y la contingencia, diferenciándose de otras sociedades que se han dado unas instituciones precisamente para absorber el impacto del acontecimiento y así garantizar su equilibrio, estabilidad y continuidad. Aunque, en realidad, nunca una sociedad logra sustraerse totalmente al impacto del acontecimiento, se trata, en efecto, de dos modos posibles de conjurarlo.

Como se recordará, en esto consistía la distinción entre sociedades "frías" y "calientes", que venía a reemplazar la antigua dicotomía entre sociedades con y sin historia. Distinción que no equivale, como se quiso alguna vez, a sociedades

2 Para la relación entre tradición bíblica, eurocentrismo y el moderno concepto de Historia resultan útiles los trabajos de Dussel, 1994 y Mignolo, 2007, también Löwith, 2007. 
progresistas y conservadoras, pues el modo de tratar con el acontecimiento en la Historia no deja de ser un modo peculiar de domesticarlo, solo que en el seno de una estructura mítica que se diferencia de la común del pensamiento salvaje: la Historia es también un mito, pero de carácter "abierto", por lo demás difícil de reconocer como mito dado que su universalización ha generado un efecto de objetividad, cuando en realidad no es más que subjetividad universalizada.

Nuestro planteamiento puede sonar anacrónico (a época del estructuralismo), pero quizá sea momento de revisar aquella polémica (sociedades frías / sociedades calientes) ya que, al parecer, hace ya tiempo nuestras sociedades se vienen "enfriando" o "cerrando", no por falta de acontecimientos, sino por la peculiar cualidad de estos. En efecto, asistimos a una proliferación inédita de acontecimientos (también inéditos), pero lo que hacemos con ellos no es ya lo que se hacía "en la época de la Historia". Es esto lo que el ya referido Hartog ha identificado como el imperio de un "régimen de historicidad presentista" o, bajo su última formulación, "una pérdida de evidencia de la historia"3.

El acontecimiento ha sido una constante de la reflexión historiográfica, desde su afirmación por la llamada historiografía positivista decimonónica (o escuela metódica), pasando por su rechazo como acto fundacional de Annales en la búsqueda de la cientificidad, hasta su negación radical por el estructuralismo: en una suerte de retorno eleatista, el conocimiento se ligaba a lo que permanece en el fondo y no a la agitación evénementielle, superficial e irrelevante, punto de partida cuando más ${ }^{4}$. Pero desde los setenta presenciamos lo que Pierre Nora denominó "la vuelta del acontecimiento", este es precisamente el título del capítulo a su cargo en el primer volumen de Hacer la historia, aquella famosa compilación, a cargo de Jacques Le Goff y el mismo Nora, que reorientó la historiografía de la última parte del siglo XX.

Para Nora, la vuelta del acontecimiento tenía relación principalmente con su "producción" y proliferación efectuada por los mass media, fenómeno que

3 Hartog, 2007 y 2014.

4 "La historia biográfica y anecdótica, que ocupa un lugar muy bajo de la escala, es una historia débil, que no contiene en sí misma su inteligibilidad, pues la alcanza solamente cuando se la transporta, en bloque, al seno de una historia más fuerte que ella [...] Y si quiere escapar al dilema, su único recurso será el salir de la historia”, Lévi-Strauss, 1990 [1962], pp. 378-379.

Nora, 1984 [1972]. 
hundía sus raíces en la última parte del siglo XIX (el caso Dreyfus) con el auge de la lógica de los periódicos: "a los mass media empezaba a corresponder el monopolio de la historia. A partir de ahora les pertenece. En nuestras sociedades contemporáneas, es mediante esos medios, y mediante ellos solos, que nos sorprende el acontecimiento; y no puede evitarnos" ${ }^{6}$. Es lo que, de modo algo irónico, llamó también "democratización de la historia", un rasgo que venía a constituir lo específico de la historia contemporánea: esa pérdida de monopolio de los acontecimientos por parte del historiador, llamativamente en el mismo momento en que los positivistas otorgaban "al acontecimiento derecho de ciudadanía no más que en un pasado inofensivo. A condición de que el presente, dominado por la tiranía del acontecimiento, viese prohibida su estancia en la historia"7. Y concluye: "es en razón de la incapacidad para dominar el acontecimiento contemporáneo, cuyas 'consecuencias' no se conocen, que, cuando los positivistas registraban inconscientemente su advenimiento para cimentar una ciencia de la historia", una disposición que, contra toda apariencia, tendría una conexión profunda con la negación del acontecimiento por la ciencia histórica del siglo XX, coincidentemente -otra vez- en el momento en que por un nuevo impulso y despliegue de los medios de comunicación masiva, la proliferación de acontecimientos, y su descontrol, se amplificaba. La cuestión del acontecimiento, o la impotencia de la historiografia frente a él, ha tenido un rol determinante -eso parece sugerir Nora- en las opciones culturales que la institución historiadora ha definido para fundar, una y otra vez, su cientificidad. ¿Qué nuevos desafíos disciplinares podría plantearnos hoy lo que parece ser una nueva vuelta del acontecimiento?

Pero en aquel paradigmático texto, Nora introducía una observación señera que marcaría el tono de la renovación historiográfica (microhistoria, historia de la vida cotidiana, historia oral, nueva historia narrativa, etc. $)^{9}$ de los setenta en adelante: "el acontecimiento es siempre revolucionario, el grano de arena en la máquina, el accidente que trastorna y pilla de improviso" ${ }^{10}$. Así el acontecimiento reingresaba -a la historiografía, pero también de modo decisivo, antes, a la filosofía y las ciencias humanas ${ }^{11}{ }^{11}$ reformulado positivamente en un pensamiento

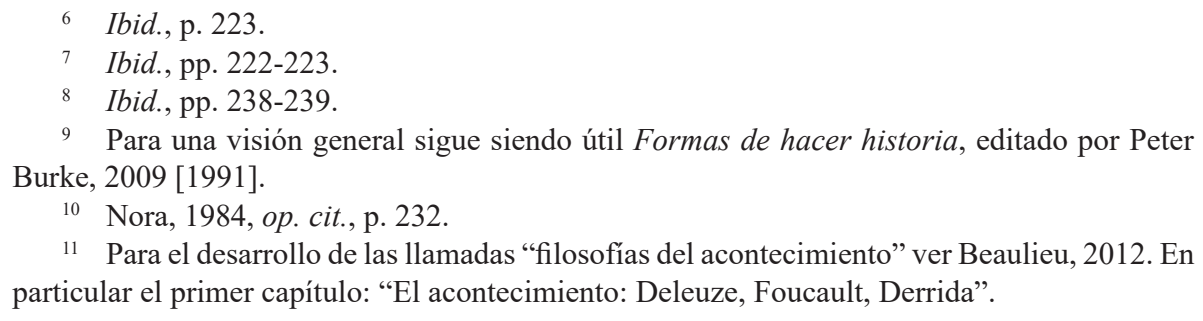

9 Para una visión general sigue siendo útil Formas de hacer historia, editado por Peter

10 Nora, 1984, op. cit., p. 232.

11 Para el desarrollo de las llamadas "filosofías del acontecimiento" ver Beaulieu, 2012. En particular el primer capítulo: "El acontecimiento: Deleuze, Foucault, Derrida". Burke, 2009 [1991]. 
cuyo campo de experiencia estaría dado principalmente por el fraude de las promesas de la modernidad y los excesos de un pensamiento social legaliforme proyectado en las catástrofes políticas del siglo XX. Podríamos decir que esa vuelta del acontecimiento fundó un pensamiento que huía de las regularidades formales, las teleologías e incluso del sentido, apostando, en cambio, por las "desviaciones" y la "emergencia". No se trataría, entonces, tanto de una "vuelta" del acontecimiento como de un "renacimiento"; usando la fórmula de François Dosse, retorna como "lo otro" o la irreductible e indomable particularidad, también como la reinterpretación constante, como aquello que una vez acaecido ya no agota más su sentido ${ }^{12}$.

Pero este renacimiento, que sigue una ruta de desarrollo intelectual en diversas variantes ${ }^{13}$, engarza y se refuerza en la actualidad con la percepción compartida de que vivimos un tiempo de fenómenos inéditos o, parafraseando a Zoltán Boldizsár Simon, una época de "cambios sin precedentes"14, acontecimientos que se relacionan con el pasado solo disociativamente, es decir, que exceden el moderno concepto de la historia como matriz para insertarlos y hacerlos inteligibles, aquel en donde, bajo diversas versiones, lo nuevo solo era asimilable como derivado de lo antiguo, lo que permitía mantener una unidad entre todo lo ya acontecido y el acontecimiento presente, unidad llamada "historia". Fenómenos como la guerra nuclear, el cambio climático, el salto tecnológico, la bioingeniería y el horizonte transhumanista, como también el empobrecimiento de vastos sectores de la población del planeta (forzando a la migración), de modo distópico han modificado la concepción del futuro quedando este desconectado del pasado: fenómenos como los aludidos no se derivarían a partir de sus precedentes (genealógica o procesualmente) sino más bien de un desarrollo fallido por los que la humanidad vislumbra, incluso, su extinción o reemplazo. La política, como campo regido por la representación humanista de la historia, se revela impotente ante este tipo de fenómenos no pudiendo ya contener la sociedad, entrando en una fase en donde los "estallidos" serían un síntoma de dicha impotencia. (A partir del moderno concepto de historia solo pueden ser entendidos como el germen del cambio político y el futuro).

12 Dosse, 2012 y 2013.

$13 \mathrm{Al}$ respecto los trabajos del ya citado François Dosse.

14 Simon, 2019. Actualmente se puede acceder de manera libre a la primera parte de su libro en el sitio de publicaciones de la Universität Bielefeld: https://pub.uni-bielefeld.de/record/2931032, desde donde hemos accedido a sus planteamientos principales. Actualmente Editorial Palinodia (Chile) prepara edición en castellano. 
En la lectura que el historiador argentino Esteban Vedia hace del libro de Simon, se desprende que "los estudios históricos en tanto siguen empeñados en la conexión pasado futuro, a contramano de un tiempo de desconexión y de novedad indomable. Nuevas perspectivas demandan nuevas formas de conceptualizar, si la narrativa histórica puede o no adecuarse a esto, está por verse" ${ }^{15}$. En la misma línea, Dipesh Chakrabarty ya había señalado (al inicio de "El clima de la historia: cuatro tesis", 2009) que la implicancia de asumir por parte de la historiografía lo que significa el calentamiento global y la categoría de "antropoceno" (la humanidad como fuerza geológica que ha modificado el sistema climático), sería el de reformular por completo lo que hasta acá llamábamos "conciencia histórica": "La disciplina de la historia existe a partir del supuesto de que nuestro pasado, presente y futuro están conectados por una cierta continuidad de la experiencia humana", pero los actuales fenómenos nos obligan a contemplar la posibilidad de "un futuro 'sin nosotros", "así nuestras habituales prácticas históricas para visualizar el tiempo [...] nos conducen a una contradicción y confusión profundas. [...] Nuestro sentido del presente, según Weisman, se ha convertido en algo profundamente destructivo en lo que se refiere a nuestro sentido general de la historia" ${ }^{\prime \prime}$.

No podemos negar una experiencia común, quizá la que define nuestra contemporaneidad: han estallado en nuestras narices, sin previo aviso, fenómenos que nos cuesta mucho trabajo explicar (incluso "creer") o cuya explicación ya no podemos seguir tramando con la Historia. Son fenómenos que nos envuelven cotidianamente, por lo que tampoco podemos acusar ya que sean un puro artilugio de los medios. Entonces, lo que vuelve no es la reducción del acontecimiento a su dimensión político-institucional del positivismo, ni tampoco el acontecimiento "producido" al que aludía Nora. Es, podríamos sostener, lo inédito en la historia.

La aparición de este tipo de acontecimientos y su efecto devastador para la historia están asociados al desarrollo industrial del capitalismo y a su proyección en ámbitos que hasta allí se habían sustraído de las lógicas de la producción, aceleración y masificación. Quizá el primer testimonio de aquel impacto en la subjetividad "moderna" fue aquella observación de Walter Benjamin (en "El narrador", 1936), respecto de que los soldados que volvían de la Primera Guerra Mundial eran incapaces ya de contar historias de guerra, y no por simple trauma, sino porque lo que allí vivieron era tan distinto a las batallas de las que habían participado antes o de las que habían escuchado de otros veteranos, que

15 Vedia, 2020, p. 143.

16 Chakrabarty, 2021, p. 9-10. 
lo vivido no pudo ser tramado con lo conocido y, por lo tanto, ya no pudieron tener ni transmitir experiencias ${ }^{17}$. ¿Qué vieron? Una guerra inédita, en donde -sabemos- entraba por primera vez el tanque, el gas y las ametralladoras (la guerra se "deshumanizaba").

Otro punto de inflexión al respecto, o toma de conciencia a nivel de las ciencias humanas, y en particular en la historiografía, lo vino a constituir el debate en torno al Holocausto y la crisis de representación asociado a lo que se denominó un "acontecimiento límite". Valga esta extensa cita de Dominick LaCapra como testimonio de la recepción del "impacto" del acontecimiento inédito en la historia:

Podemos definir como acontecimiento límite aquel que supera la capacidad imaginativa de concebirlo o anticiparlo. Antes de que ocurriera no fue -acaso no pudo serlo- previsto ni imaginado, y no sabemos a ciencia cierta qué es verosímil o plausible en ese contexto. En todo caso, hubo una resistencia extrema a vislumbrar su posibilidad. De allí que este acontecimiento (o serie de acontecimientos) deba necesariamente ser traumático o traumatizante, y que lo que pide a gritos una explicación sea la no traumatización de quien lo ha experimentado. Incluso después de experimentado, un acontecimiento de esta clase pone a prueba y posiblemente supera la imaginación, incluso la de quienes no lo experimentaron directamente (los que no estuvieron allí). Los hechos pueden superar nuestra facultad imaginativa y hasta parecer increíbles ${ }^{18}$.

17 "Con la Guerra Mundial comenzó a hacerse evidente un proceso que aún no se ha detenido. ¿No se notó acaso que la gente volvía enmudecida del campo de batalla? En lugar de retornar más ricos en experiencias comunicables, volvían empobrecidos. Todo aquello que diez años más tarde se vertió en una marea de libros de guerra, nada tenía que ver con experiencias que se transmiten de boca en boca", Benjamin, 2001, p. 112.

18 LaCapra, 2006, p. 181. Con todo se podría pensar que las reflexiones de LaCapara quedan en el orden de un historiador dedicado más bien a cuestiones de orden teóricas, más un filósofo que un historiador, como suele acotarse en ciertos debates despectivamente. Pero el impacto del acontecimiento límite está también atestiguado por historiadores de oficio. Valga al respecto el siguiente fragmento de una entrevista a Eric Hobsbawm: “A. Spire: En la apertura de la Historia del siglo XX, cita a Primo Levi. [...] Naturalmente, pensé que usted hablaría de los campos, pero no hay ni una palabra sobre el tema [...] ¿Cómo es posible que la situación del hombre de los campos no haya merecido su atención?

E. Hobsbawm: Precisamente es la cita de Primo Levi lo que explica eso. Lo que es posible hacer es el análisis, la génesis del fenómeno del genocidio, cómo fue organizado, cuáles fueron sus motivaciones, etc. Pero en mi opinión, el hecho del genocidio y la misma experiencia de los campos de concentración superan las palabras. Intencionadamente, no he querido describir esas experiencias, esos acontecimientos inexpresables, y en el libro he procurado, en la medida de lo posible, mantenerlo. Pero la Shoah, las matanzas de decenas de millones de personas en las 
Cuando hablamos de "acontecimiento inédito" queremos aludir a un tipo de acontecimientos que solo aparentemente son similares a otros antiguos, pero que, analizados de cerca, exceden los rasgos de lo conocido, más aún, el registro de lo conocido hasta pareciera inducirnos a su incomprensión: pudiera ser que ya la historia no sea la que nos ayude a echar luz sobre el presente, sino justamente la que no nos deja entrar en su especificidad. Tratar de comprender "históricamente" un acontecimiento -ligarlo a lo que conocemos del pasado-hoy implicaría, más que antes en la modernidad, no asir justamente el componente de novedad (lo otro) que, no obstante, lo histórico nos permite intuir. He aquí la paradoja que se instala en nuestra actual "conciencia histórica".

Pero - para colmo de paradojas- este último rasgo no sería del todo nuevo o, quizá, lo propiamente nuevo sea lo expansiva de esta-si no toma de concienciaintuición de que el pasado ya no puede ayudarnos a pensar el futuro, pues desde el siglo XIX se supo que "el pasado ya no ilumina el porvenir y el espíritu humano camina entre tinieblas" (rescatando la cita de Tocqueville a la que volviera de modo ilustrativo Hannah Arendt). Lo novedoso hoy sería la extraordinaria aceleración con la que caduca nuestra experiencia o, desde otra perspectiva, la velocidad con la que emerge lo inédito. Sin embargo, lo particular de nuestra hora sería una entrada de lo "inédito amenazador" (epidemias, catástrofes, "estallidos", etc. $)^{19}$, distinto de lo "inédito emergente" de las filosofías del acontecimiento, como de la nueva historia en que, de alguna manera, lo emergente equivalía a unas deseables - por su potencial emancipador- desviaciones de lo regular y ya determinado, alimentando un optimismo histórico solo equiparable al de la idea de progreso, pero sin su carácter procesal. "Progreso sin progreso".

Como se sigue de los razonamientos de Nora arriba expuestos, como de nuestra caracterización del actual retorno del acontecimiento, debemos ser capaces de vislumbrar qué opciones culturales está tomando la institución historiadora frente a esta radical insubordinación del acontecimiento, aunque esa opción fuera la pura negación. Es frente a este tipo de cuestionamiento que se ha organizado el presente dossier.

guerras -en el fondo, incluso si se trata de las dos guerras mundiales-, todo eso, sobrepasa en mi opinión las fuerzas del historiador", Hobsbawm, 2004, p. 42.

19 Sobre este punto en particular se puede consultar la entrevista que realicé a Hartog el año 2013, titulada "François Hartog: la historia en un tiempo catastrófico", publicada en esta misma revista, Aravena, 2014. 


\section{Bibliografia}

Aravena, Pablo, "François Hartog: la historia en un tiempo catastrófico", Cuadernos de Historia, $\mathrm{N}^{\circ} 41$, Santiago, Departamento de Ciencias Históricas, Universidad de Chile, 2014, pp. 227-234.

Beaulieu, Alain, Cuerpo y acontecimiento. La estética de Gilles Deleuze, Buenos Aires, Letra Viva, 2012.

Benjamin, Walter, “El Narrador”, en Walter Benjamin, Para una Crítica de la Violencia y Otros Ensayos. Iluminaciones IV, Madrid, Taurus, 2001, pp. 111-134.

Burke, Peter, Formas de hacer historia, Madrid, Alianza, 2009.

Chakrabarty, Dipesh, Clima y Capital. La vida bajo el antropoceno, Viña del Mar, Mímesis, 2021.

Dosse, François, "El acontecimiento histórico entre Esfinge y Fénix", Historia y Grafía, México, Universidad Iberoamericana, $N^{\circ} 41,2013$, pp. 13-42.

DosSE, FrançoIs, El giro reflexivo de la historia. Recorridos epistemológicos y atención a las singularidades, Santiago, Ediciones Universidad Finis Terrae, 2012.

Dussel, EnRIQue, 1492 El encubrimiento del otro: hacia el origen del "mito de la Modernidad", Bolivia, Plural, 1994.

Hartog, François, "La temporalización del tiempo: un largo recorrido", en Jaques André (dir.), Los relatos del tiempo, Buenos Aires, Nueva Visión, 2011.

Hartog, François, Creer en la historia, Santiago, Ediciones de la Universidad Finis Terrae, 2014.

Hartog, François, Regímenes de historicidad. Presentismo y experiencias del tiempo, México, Universidad Iberoamericana, Departamento de Historia, 2007.

Hobsbawm, Eric, El optimismo de la voluntad. Conversación con Antoine Spire, Barcelona, Paidós Asterisco, 2004.

LaCapra, Dominick, Historia en tránsito. Experiencia, identidad, teoría crítica, Buenos Aires, Fondo de Cultura Económica, 2006.

LÉvi-Strauss, Claude, El pensamiento salvaje, México D. F., Fondo de Cultura Económica, 1990.

LöwITH, KARL, Historia del mundo y salvación. Los presupuestos teológicos de la filosofía de la historia, Buenos Aires, Katz, 2007.

Mignolo, Walter, La idea de América Latina. La herida colonial y la opción decolonial, Barcelona, Gedisa, 2007.

Nora, Pierre, "La vuelta del acontecimiento", en Jaques Le Goff y Pierre Nora (dirs.), Hacer la historia, Volumen I, Barcelona, Editorial Laia, 1984, pp. 221-239.

Simon, Zoltán, History in Times of Unprecedented Change. A Theory for the 21st Century, London, Bloomsbury, 2019.

Vedia, EsteBAn, “Zóltan Boldizsár Simon, History in Times of Unprecedented Change. A Theory for the 21st Century. London: Bloomsbury, 2019, 209 pp.”, Historiografias, $\mathrm{N}^{\circ}$ 20, Zaragoza, 2020, pp. 143-148. 
